\title{
Impaired cardiac autonomic control relates to disease severity in pulmonary hypertension
}

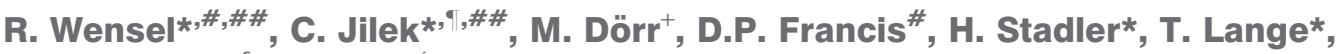 \\ F. Blumberg ${ }^{\star, \S}$, C. Opitz ${ }^{\dagger}$, M. Pfeifer ${ }^{\star \star \star \star}$ and R. Ewert ${ }^{+}$
}

ABSTRACT: Pulmonary arterial hypertension (PAH) results in chronic right heart failure, which is associated with an increase in sympathetic tone. This may adversely affect cardiac autonomic control. We investigated the changes in cardiac autonomic nervous activity in relation to disease severity in patients with PAH.

In 48 patients with PAH (median World Health Organization class III, pulmonary artery pressure $52 \pm 14 \mathrm{mmHg}$, pulmonary vascular resistance $1,202 \pm 718 \mathrm{dyn} \cdot \mathrm{s} \cdot \mathrm{cm}^{-5}$, cardiac index $2.0 \pm 0.8 \mathrm{~L} \cdot \mathrm{min}^{-1} \cdot \mathrm{m}^{-2}$ ) and 41 controls, cardiac autonomic nervous activity was evaluated by measurement of heart rate variability (HRV) and baroreflex sensitivity. All patients underwent cardiopulmonary exercise testing (peak oxygen uptake $13.2 \pm 5.1 \mathrm{~mL} \cdot \mathrm{kg}^{-1} \cdot \mathrm{min}^{-1}$, minute ventilation/carbon dioxide production slope $47 \pm 16$ ).

In patients with PAH, spectral power of HRV was reduced in the high-frequency $(239 \pm 64$ versus $\left.563 \pm 167 \mathrm{~ms}^{2}\right)$, low-frequency $\left(245 \pm 58\right.$ versus $\left.599 \pm 219 \mathrm{~ms}^{2}\right)$ and very low-frequency bands $\left(510 \pm 149\right.$ versus $1106 \pm 598 \mathrm{~ms}^{2}$; all $\left.\mathrm{p}<0.05\right)$. Baroreflex sensitivity was also blunted $(5.8 \pm 0.6$ versus $\left.13.9 \pm 1.2 \mathrm{~ms} \cdot \mathrm{mmHg}^{-1} ; \mathrm{p}<0.01\right)$. The reduction in high-frequency $(r=0.3, p=0.04)$ and lowfrequency $(r=0.33, p=0.02)$ spectral power and baroreflex sensitivity $(r=0.46, p<0.01)$ was related to the reduction in peak oxygen uptake.

Patients with PAH have a marked alteration in cardiac autonomic control that is related to exercise capacity and may, therefore, serve as an additional marker of disease severity.

KEYWORDS: Exercise capacity, heart autonomic system, heart rate variability, pulmonary hypertension

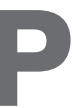

ulmonary arterial hypertension $(\mathrm{PAH})$ is a rapidly progressive disease leading to right heart failure and death [1]. The increasing availability of medical therapy has substantially improved symptoms and prognosis [1]. In the decision process of initiation and escalation of treatment, risk stratification of the patients plays a major role. In this regard there is increasing awareness that chronic right heart failure is a syndrome that affects many organ systems including the neuroendocrine system [24] and renal function [5], and that these secondary changes are a marker of disease severity, progression and prognosis. Current evaluation of disease severity is based on measurement of resting haemodynamics [6], exercise capacity [7] and neurohormonal markers [2-4].
$\mathrm{PAH}$ is associated with in increased sympathetic tone as assessed by muscle sympathetic nerve activity, whereas there are conflicting data regarding plasma catecholamine levels [8, 9]. The increased sympathetic tone seems to be related to the haemodynamic abnormalities.

In left heart failure, chronic sympathetic over activity leads to an impairment of cardiac autonomic nervous activity (CANA) with reduced baroreflex sensitivity (BRS) and altered heart rate variability (HRV) [10]. The altered autonomic tone is associated with, and possibly also a mediator of, impaired prognosis in these patients [11-13].

In animal models of monocrotaline-induced pulmonary hypertension, reduction in spectral

\section{AFFILIATIONS}

*Dept of Internal Medicine II, Universtätsklinikum Regensburg, Regensburg,

"Deutsches Herzzentrum München, Klinik der Technischen Universität München, Munich,

${ }^{+}$Dept of Internal Medicine B, ErnstMoritz-Arndt University Greifswald Greifswald,

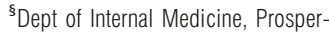
Hospital, Recklinghausen,

${ }^{f}$ Dept of Internal Medicine/ Cardiology, DRK Kliniken Berlin Köpenick, Berlin, and

${ }^{* \star}$ Center for Pneumonology, Donaustauf Hospital, Donaustauf, Germany.

\#International Centre for Circulatory Health, National Heart and Lung Institute, Imperial College London, London, UK.

\#\#These authors are joint first authors.

CORRESPONDENCE

R. Wensel

International Centre for Circulatory Health

St Mary's Hospital and Imperial

College

59 North Wharf Rd

London

W2 1LA

UK

E-mail: rolandwensel@web.de

Received:

Sept 242008

Accepted after revision:

April 242009

First published online:

May 142009

European Respiratory Journal

Print ISSN 0903-1936

Online ISSN 1399-3003

For editorial comments see page 792 . 
power of HRV has been observed [14]. Changes in spectral distribution of HRV with reduction of low-frequency (LF) and high-frequency (HF) power [15], and increased LF versus HF spectral power ratio (LF/HF ratio) have been described in patients with $\mathrm{PAH}$ [16]. These changes were partially reversible on treatment with subcutaneous treprostinil. This led to the hypothesis that in patients with PAH there is an alteration in cardiac autonomic nervous activity, which may also relate to disease severity.

Therefore, we set out to characterise the cardiac autonomic nervous activity and its relationship to exercise capacity as a marker of disease severity in patients with symptomatic PAH.

\section{METHODS}

\section{Patients}

We studied 48 patients with PAH; 36 with idiopathic PAH, four with $\mathrm{PAH}$ associated with congenital heart defects and eight with PAH associated with connective tissue disease (13 males, aged $51 \pm 12$ yrs). Patients were in median World Health Organization class III. Haemodynamic assessment showed a marked degree of pulmonary hypertension and reduced cardiac index (mean pulmonary artery pressure $52 \pm 14 \mathrm{mmHg}$, pulmonary vascular resistance $1,202 \pm 718 \mathrm{dyn} \cdot \mathrm{s} \cdot \mathrm{cm}^{-5}$ and cardiac index $\left.2.0 \pm 0.8 \mathrm{~L} \cdot \mathrm{min}^{-1} \cdot \mathrm{m}^{-2}\right)$. Chronic medication included endothelin-1 receptor antagonists $(n=29)$, phosphodiesterase type 5 inhibitors $(n=2)$, inhaled iloprost $(n=7)$, intravenous iloprost $(n=5)$, oral beraprost $(n=4)$, calcium channel antagonists $(n=2)$, oral anticoagulation $(n=37)$, spironolactone $(n=16)$, loop diuretics $(n=32)$ and digoxin $(n=3)$.

\section{Subjects}

In total, 41 age and sex matched healthy individuals (12 males, $48 \pm 13$ yrs of age, heart rate $69 \pm 10$ beats $\cdot \mathrm{min}^{-1}$ ) served as a control group for measurements of HRV and BRS. All subjects had no history of a medical illness, a normal physical examination and none of them were taking any medication.

The study complied with the Declaration of Helsinki and was been approved by the institutional ethics committee (Medical Faculty of the University of Regensburg, Regensburg, Germany). Informed consent was obtained from all patients.

\section{Measurements}

\section{Cardiopulmonary exercise testing}

All patients underwent cardiopulmonary exercise testing (CPET). The exercise test was performed on a treadmill in 26 patients and with a cycle ergometer in 22 patients. The modified Naughton protocol for treadmill exercise testing was used. Exercise testing with the use of a cycle ergometer (ER 900; Jäger, Würzburg, Würzburg, Germany) was started at $20 \mathrm{~W}$ with a stepwise increment of $16 \mathrm{~W} \cdot \mathrm{min}^{-1}$. Oxygen uptake $\left(V^{\prime} \mathrm{O}_{2}\right)$, carbon dioxide production $\left(V^{\prime} \mathrm{CO}_{2}\right)$, instantaneous expiratory gas concentrations throughout the respiratory cycle and minute ventilation $\left(V^{\prime} \mathrm{E}\right)$ were measured continuously on a breath-by-breath basis (Oxycon; Jaeger-Viasys, Germany).

Peak $V^{\prime} \mathrm{O}_{2}$ was defined as the oxygen uptake measured at peak exercise, which always occurred well beyond the anaerobic threshold. $V^{\prime} \mathrm{O}_{2}$ at the gas exchange-anaerobic threshold was detected with the V-slope method [17], supplemented by the simultaneous observation of end-tidal gas concentrations.
Pulmonary gas exchange was assessed by the $V^{\prime} \mathrm{E} / V^{\prime} \mathrm{CO}_{2}$ ratio and the slope of this relationship on exercise, as reported previously [18]. Heart rate and blood pressure (using sphygmomanometer) were measured at rest and during each stage of exercise.

\section{HRV and BRS}

Patients and controls were studied between 13:00 and 17:00 under standardised conditions, in a quiet air-conditioned room. Before data acquisition, all patients were in the fasting state for at least $2 \mathrm{~h}$ and were not allowed to smoke or drink beverages containing alcohol or caffeine for $24 \mathrm{~h}$. Subjects rested in the supine position for $15 \mathrm{~min}$ and then underwent two successive investigations: one continuous 20-min recording at rest and one 5-min recording during controlled breathing at $0.1 \mathrm{~Hz}$ by following a sinusoidal visual and auditory signal [19]. During each investigation, R-R interval, blood pressure (BP) and respiration were recorded. Procedures were performed in the same order in all subjects. BP was measured by a tonometric device (CBM 7000; Colin Medical Instruments, San Antonio, TX, USA), with the pressure probe placed over the right radial artery and the wrist resting comfortably at the level of the heart. ECG was acquired from the limb lead with the largest $\mathrm{R}$ wave (Sirekust732; Siemens AG, Munich, Germany). Respiratory rate was measured by thoracic impedance (Sirekust732; Siemens AG). All data were sampled at $1,000 \mathrm{~Hz}$ on a computer using an analogue-to-digital converter (National Instruments, Austin, TX, USA). Readings were saved and analysed off-line with custom designed software. The program measured $R-R$ intervals and beat-to-beat systolic pressure while ectopic beats were excluded by linear interpolation [19].

\section{HRV}

HRV analysis was made on 20-min ECG recordings and timeand frequency-domain indexes were calculated using our laboratory's software. The following frequency-domain indices were assessed: 1) very low-frequency (VLF) power $(0.0033$ $0.04 \mathrm{~Hz})$; 2) LF power $(0.04-0.15 \mathrm{~Hz})$; and 3$) \mathrm{HF}$ power $(0.15-$ $0.4 \mathrm{~Hz})$.

\section{$B R S$}

For BRS assessment, we measured average amplitudes of oscillations in the R-R interval and systolic blood pressure (SBP). BRS was assessed noninvasively using the following methods [20].

\section{BRS frequency domain index}

The square root of the ratio between RR and SBP power spectral analysis was performed on the R-R interval and SBP data using an autoregressive algorithm $[19,21]$. The $\alpha$-index $(\alpha \mathrm{LF})$ was calculated in the presence of an adequate coherence $(>0.5)$ between the R-R interval and SBP as assessed by crossspectral analysis.

\section{BRS sequence method}

The average value of the individual linear correlation slopes between RR and SBP during sequences in which RR and SBP concurrently increased or decreased over three or more beats. The minimum change required was $1 \mathrm{mmHg}$ for SBP and $4 \mathrm{~ms}$ 


\begin{tabular}{|c|c|c|}
\hline TABLE 1 & \multicolumn{2}{|c|}{$\begin{array}{l}\text { Results of cardiopulmonary exercise testing of } \\
\text { pulmonary arterial hypertension patients }\end{array}$} \\
\hline \multicolumn{3}{|l|}{ Parameter } \\
\hline \multicolumn{2}{|c|}{ Exercise duration $\mathbf{s}$} & $384 \pm 33$ \\
\hline \multicolumn{2}{|c|}{ SBP rest $\mathrm{mmHg}$} & $121 \pm 3$ \\
\hline \multicolumn{2}{|c|}{ DBP rest $\mathrm{mmHg}$} & $76 \pm 2$ \\
\hline \multicolumn{2}{|c|}{ SBP peak exercise $\mathrm{mmHg}$} & $150 \pm 6$ \\
\hline \multicolumn{2}{|c|}{ DBP peak exercise $\mathrm{mmHg}$} & $83 \pm 3$ \\
\hline \multicolumn{2}{|c|}{ Oxygen uptake at anaerobic threshold ${ }^{\#} \mathrm{~mL} \cdot \mathrm{kg}^{-1} \cdot \mathrm{min}^{-1}$} & $10.4 \pm 1.1$ \\
\hline \multicolumn{2}{|c|}{ Peak oxygen uptake $\mathrm{mL} \cdot \mathrm{kg}^{-1} \cdot \mathrm{min}^{-1}$} & $13.2 \pm 5.1$ \\
\hline \multicolumn{2}{|c|}{ RER at peak exercise } & $1.03 \pm 0.05$ \\
\hline \multicolumn{2}{|c|}{$V^{\prime} \mathrm{E} / \mathrm{V}^{\prime} \mathrm{CO}_{2}$ rest } & $46 \pm 1$ \\
\hline \multicolumn{2}{|c|}{$P \mathrm{ET}, \mathrm{CO}_{2}$ rest $\mathrm{mmHg}$} & $26.1 \pm 0.7$ \\
\hline \multicolumn{2}{|c|}{$V^{\prime} \mathrm{E}^{\prime} \mathrm{V}^{\prime} \mathrm{CO}_{2}$ slope } & $47 \pm 16$ \\
\hline \multicolumn{2}{|c|}{$\mathrm{Sa}, \mathrm{O}_{2}$ rest $\%$} & $92 \pm 1$ \\
\hline \multicolumn{2}{|c|}{$\mathrm{Sa}, \mathrm{O}_{2}$ peak exercise $\%$} & $87 \pm 1$ \\
\hline
\end{tabular}

Data are presented as mean \pm SD. SBP: systolic blood pressure; DBP: diastolic blood pressure; RER: respiratory exchange ratio; $V^{\prime} E$ : minute ventilation; $V^{\prime} \mathrm{CO}_{2}$ : carbon dioxide production; $\mathrm{PET}, \mathrm{CO}_{2}$ : end-tidal carbon dioxide tension; $\mathrm{Sa}_{1} \mathrm{O}_{2}$ : arterial oxygen saturation. ${ }^{\#:} n=33 ;{ }^{\circ}: n=40$.

for $\mathrm{R}-\mathrm{R}$ interval. The regression slope was calculated in those sequences with correlation coefficients of $>0.8$.

\section{BRS during controlled breathing}

The ratio of the average amplitude of oscillation in $R R$ to the average amplitude of oscillations in SBP during a 5-min interval of controlled breathing [19]. The resultant RR and SBP signals were processed with a simple time-domain digital filter to extract the signal component at the frequency of interest $(0.1 \mathrm{~Hz})$.

\section{Statistical analysis}

Data are presented as mean \pm SD. Indices with skewed distribution were log-transformed. Data were analysed with Sigma Stat 3.0 (SPSS, Chicago, IL, USA). Differences between patients and controls were analysed with an unpaired t-test or the Mann-Whitney test if data did not follow a normal distribution. The relationship between two variables was tested using the Pearson correlation. A p-value $<0.05$ was considered significant.

\section{RESULTS}

\section{CPET}

The CPET results of the patients with PAH are presented in table 1 . In 15 patients the $V^{\prime} \mathrm{O}_{2}$ at the anaerobic threshold could not be determined because of very low exercise capacity. Seven patients showed a pattern of pulmonary gas exchange consistent with a right-to-left shunt during exercise [22], which resulted in a nonlinear $V^{\prime} \mathrm{E} / V^{\prime} \mathrm{CO}_{2}$ relationship. In these patients, the $V^{\prime} \mathrm{E} / V^{\prime} \mathrm{CO}_{2}$ slope was not calculated.

\section{HRV and BRS}

Patients with PAH showed a reduction in total power of HRV (962 versus 1,938 $\mathrm{ms}^{2} ; \mathrm{p}<0.001$ ) analysed in all spectral bands when compared to healthy controls (fig. 1). However, there was no significant difference in the LF/HF ratio (1.28 versus 1.04; $\mathrm{p}=0.19)$. As shown in figure 2 , BRS was consistently decreased in patients with $\mathrm{PAH}$.

\section{Relationship of HRV to exercise capacity and pulmonary gas exchange}

The reduction of both HF and LF power was associated with progressive impairment of exercise capacity as assessed by peak $V^{\prime} \mathrm{O}_{2}$ (fig. 3). However, there was no significant correlation with the impairment of ventilator efficiency (logHF versus $V^{\prime} \mathrm{E} / V^{\prime} \mathrm{CO}_{2}$ slope $\mathrm{r}=-0.13, \mathrm{p}=0.4 ; \log \mathrm{LF}$ versus $V^{\prime} \mathrm{E} / V^{\prime} \mathrm{CO}_{2}$ slope $\mathrm{r}=-0.22, \mathrm{p}=0.14)$.

\section{Relationship of BRS to exercise capacity and pulmonary gas exchange}

BRS, as assessed by the controlled breathing method (fig. 4), and the $\alpha$ LF-index revealed a significant correlation with peak $V^{\prime} \mathrm{O}_{2}(\mathrm{r}=0.46, \mathrm{p}<0.01$ and $\mathrm{r}=0.36, \mathrm{p}<0.05$, respectively). No significant correlation with the $V^{\prime} \mathrm{E} / V^{\prime} \mathrm{CO}_{2}$ slope was found $(\mathrm{r}=-0.13, \mathrm{p}=0.4)$
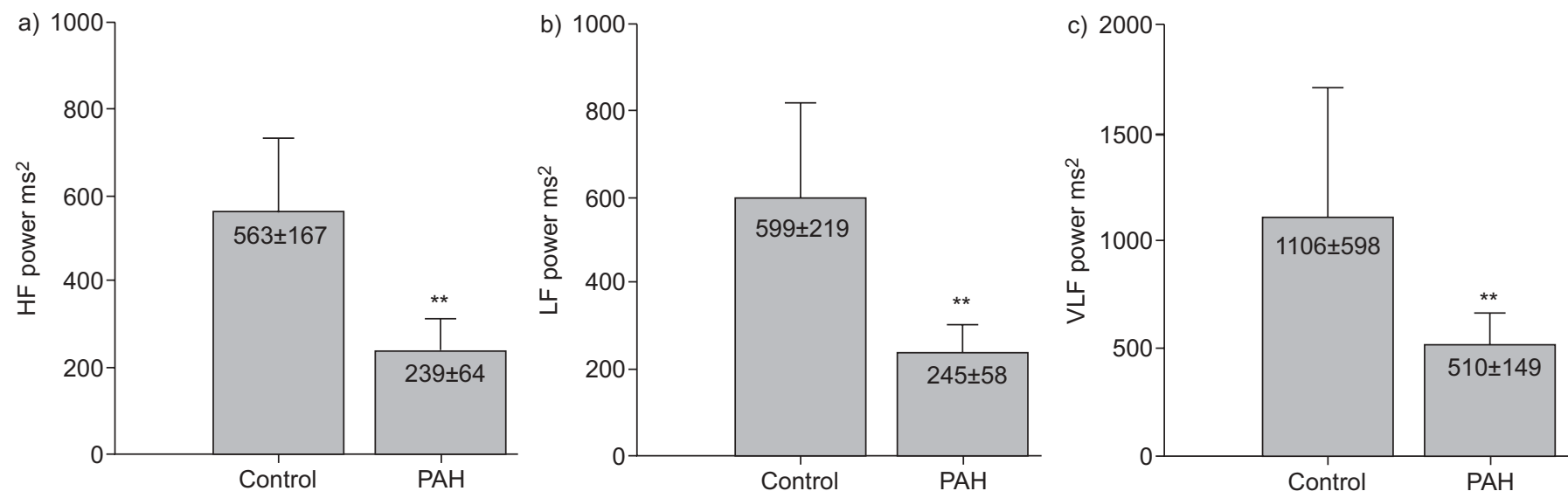

FIGURE 1. a) High-frequency (HF), b) low-frequency (LF) and c) very low-frequency (VLF) spectral power in patients with pulmonary arterial hypertension (PAH) versus controls. ${ }^{*}$ : $p<0.01$ 

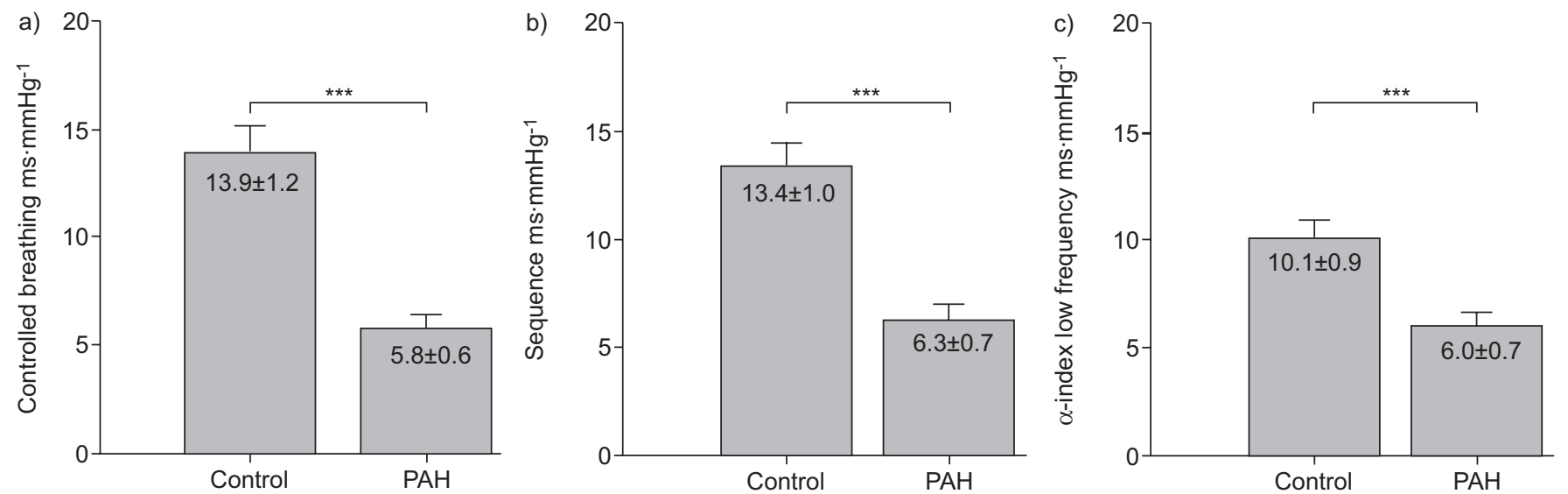

FIGURE 2. Baroreflex sensitivity as assessed by a) controlled breathing, b) sequence method and c) $\alpha$-index in patients with pulmonary arterial hypertension (PAH) versus controls. ${ }^{* *}: \mathrm{p}<0.001$.

\section{DISCUSSION}

PAH results in chronic right heart failure which is not just a condition of pure haemodynamic failure but a syndrome affecting many organ systems.

Increased sympathetic activity, as is evident from increased systemic vascular resistance [23] or direct measurement of sympathetic nervous activity [8], may have detrimental effects on disease progression that parallels the effects seen in chronic left heart failure. However, little is known about such consequences of sympathetic over activity in PAH.

Our study shows that in patients with $\mathrm{PAH}$, chronic sympathetic over activity leads to profound changes in cardiac autonomous regulation and that these changes relate to disease severity.

\section{HRV}

We assessed HRV based on 20-min resting ECG recordings under standardised external conditions. This duration of measurement allows ample time for the frequencies of interest. Compared to 24-h recording, this has the advantage of eliminating the variation in physical activity between patients, which inevitably occurs during unrestricted and unobserved behaviour.

We observed a marked reduction in the total power of HRV with similar reduction of HF, LF and VLF power compared with healthy controls.

Since HRV in the HF band is mediated by the vagal activity, our findings indicate vagal withdrawal, which can be explained by the underlying cardiac failure. The observed reduction in the LF power, which is mainly regulated by sympathetic innervation, may be surprising at first. Sympathetic stimulation that results from physiological changes (i.e. orthostasis, exercise or cold exposure) or acute and subacute disease (i.e. post-myocardial infarction) increases LF power compared to total power and HF power [11, 20]. In contrast, chronic sympathetic stimulation, as in chronic left heart failure, leads to a reduction in LF power indicating a reduced susceptibility to respond to changes in sympathetic tone $[12,13]$. This suggests that the reduction in LF power in $\mathrm{PAH}$ patients results from chronic sympathetic activation, which has been reported previously [8]. The concomitant changes in HF and LF power explain the absence of a significant change in the LF/HF ratio compared with healthy controls. FAUCHIER et al. [15] described much higher LF/HF for patients with $\mathrm{PAH}$ as well as for normal individuals. However, they used 24-h ECG recordings and their finding might be
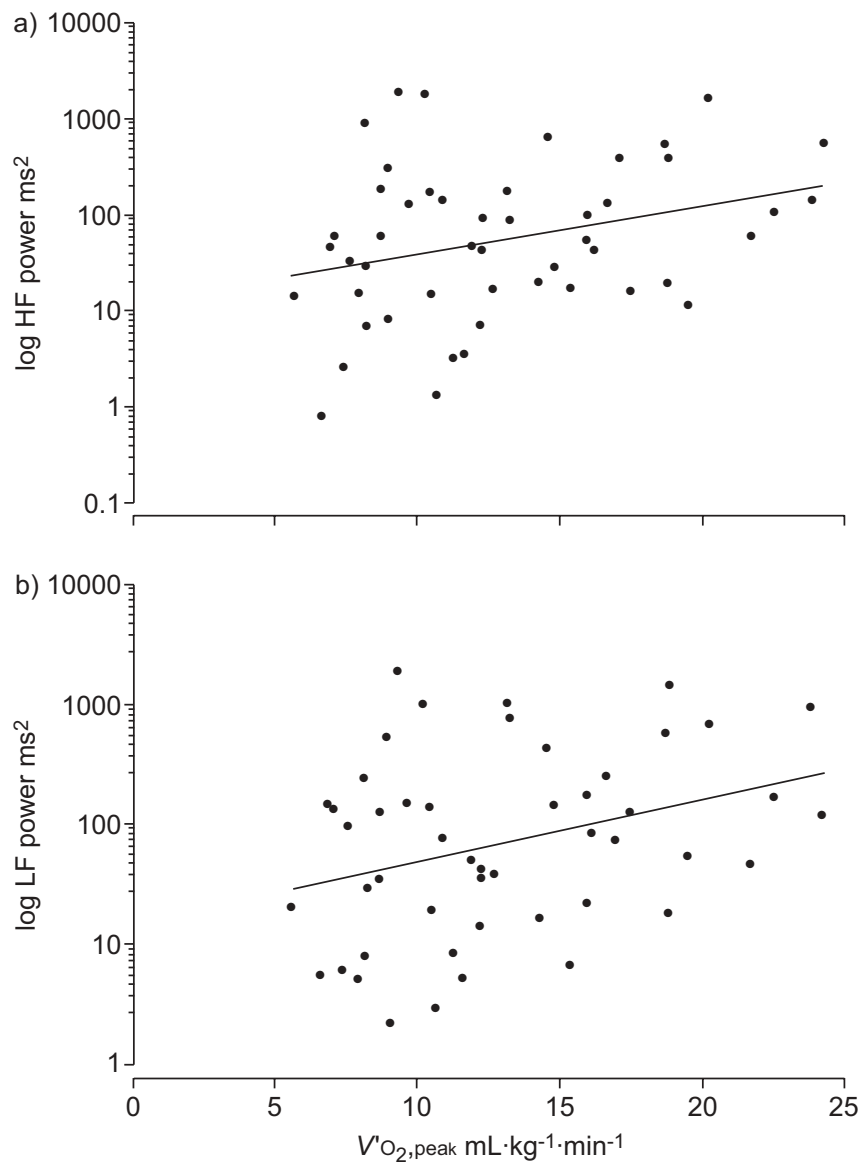

FIGURE 3. Relationship between spectral power in a) high frequency (HF) and b) low frequency (LF) power and peak oxygen uptake $\left(V^{\prime} \mathrm{O}_{2}\right.$,peak $)$. a) $r=0.29$, $p=0.04$. b) $r=0.31, p=0.03$. 


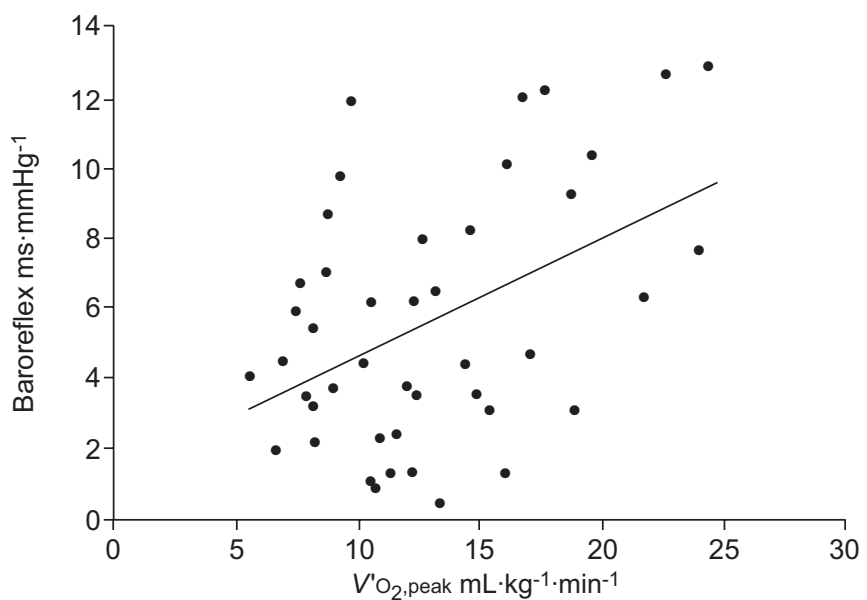

FIGURE 4. Relationship between baroreflex sensitivity, as measured by the controlled breathing method, and peak oxygen uptake $\left(V^{\prime} \mathrm{O}_{2}\right.$, peak). $r=0.46, p<0.01$.

explained by higher physical activity during the sampling period.

\section{BRS}

We used three different noninvasive methods to assess BRS and consistently observed a marked reduction in BRS. These results fit perfectly with the observed changes in HRV.

First, the baroreflex consists of a reciprocal change in heart rate and systemic vascular resistance in response to spontaneous changes in arterial BP [24]. The afferent pathway involves parasympathetic fibres of the vagal and glossopharyngeal nerve. The efferent pathway involves both sympathetic (reduced when BP rises) and vagal outflow (increased when $\mathrm{BP}$ rises). Therefore, a blunted BRS contributes to reduced vagal outflow and unrestricted sympathetic outflow to the heart, which is reflected by the changes in LF and HF power.

Secondly, the LF band has been suggested to reflect the baroreflex induced changes in heart rate and, therefore, reduced BRS results in reduced LF power $[25,26]$.

\section{What is the cause of altered cardiac autonomous activity in PAH?}

Chronic sympathetic over activity, which has been described in $\mathrm{PAH}$, is a likely cause of the change in autonomous control. The data from CIARKA et al. [9] suggest that sympathetic activity is directly related to the severity of the underlying haemodynamic failure with increased right atrial pressures and low cardiac output in PAH. However, the changes in cardiac autonomic nervous activity observed in our study parallel those seen in patients with chronic left heart failure $[10,12]$ and other chronic cardiac conditions associated with relative circulatory failure, for example, in adults with congenital heart defects $[27,28]$. This suggests that the chronic autonomic imbalance, and its effects on cardiac autonomous nervous activity, is a nonspecific response to chronic circulatory/heart failure regardless of its origin. Furthermore, data from patients with predominant left heart failure also suggest that peripheral changes occurring secondary to the underlying haemodynamic disorder are major contributors to increased sympathetic activity [21, 29].
In chronic left heart failure, peripheral blockade of sympathetic over activity improves cardiac function, symptoms and prognosis and is, therefore, an integral part of medical therapy [30]. Is there a role for such therapy in patients with PAH? Currently, no controlled data exists on this issue; nevertheless, $\beta$-blockade is usually poorly tolerated in PAH and may lead to acute decompensation of right heart failure (i.e. overt right heart failure with fluid retention and symptoms at rest), even when administered at minute doses (M.M. Hoeper, Dept of Respiratory Medicine, Hannover Medical School, Hannover, Germany; personal communication). This difference to left heart failure may be explained by the chronic unrelieved pressure overload of the right ventricle which requires chronic inotropic support by sympathetic stimulation. However, this may change with further improvements in medical therapy aiming to reverse pulmonary vasculopathy.

\section{CANA in relation to exercise capacity and pulmonary gas exchange}

We found a severely reduced peak $V^{\prime} \mathrm{O}_{2}$ and ventilatory efficiency (assessed by the $V^{\prime} \mathrm{E} / V^{\prime} \mathrm{CO}_{2}$ ratio or $V^{\prime} \mathrm{E} / V^{\prime} \mathrm{CO}_{2}$ slope) in our patients, which is consistent with previous reports of PAH [23, 31, 32]. In our study, the alteration in cardiac autonomic modulation was related to disease severity as measured by peak $V^{\prime} \mathrm{O}_{2}$. Thus, it can be concluded that cardiac autonomous control is related to disease severity in PAH. However, no significant relationship between parameters of ventilatory efficiency and CANA was found. This is a surprising finding as the $V^{\prime} \mathrm{E} / V^{\prime} \mathrm{CO}_{2}$ ratio and slope have consistently been shown to be increased in PAH [23, 31, 32]. A potential explanation to this is that the $V^{\prime} \mathrm{E} / V^{\prime} \mathrm{CO}_{2}$ slope depends on the patient's effort, if all exercise data are included in the regression analysis. In fact, the mean peak respiratory exchange ratio was relatively low in our study, which might imply that $V^{\prime} \mathrm{E} / V^{\prime} \mathrm{CO}_{2}$ slope was measured incorrectly in some patients. This would confound the relationship between the $V^{\prime} \mathrm{E} / V^{\prime} \mathrm{CO}_{2}$ slope and CANA.

Measurements of HRV and BRS are noninvasive and do not inconvenience the patient. Therefore, they can be obtained and repeated at any stage of disease. This implies a potential role of these measurements in risk stratification and treatment effects in PAH. However, this requires further investigation.

\section{Study limitations}

A potential limitation of our study was that we did not directly measure sympathetic outflow. Direct measurement by muscle sympathetic nerve activity, which is a difficult and sometimes uncomfortable procedure, is recognised to show increased sympathetic activity that relates to disease severity in $\mathrm{PAH}$. Potentially more accessible indices, such as plasma catecholamine levels, are unfortunately not reliable at reflecting sympathetic status in PAH [8]. However, our study did not aim to measure sympathetic activity, which has been shown to be increased in PAH previously [8, 9], but to investigate the effect on cardiac autonomous control and its relationship to disease severity. Therefore, we took a pragmatic approach of obtaining measurements using methods that patients found acceptable, and were likely to reflect cardiac autonomic nerve function. 
The patients in our study had been suffering from $\mathrm{PAH}$, including idiopathic $\mathrm{PAH}, \mathrm{PAH}$ related to connective tissue disease and PAH related to congenital heart defects. Therefore, it is possible that our results have been confounded by the underlying disease (i.e. connective tissue disease). However, the presence of PAH appears to have a major adverse effect on the symptoms and prognosis of such patients [33]. In fact, any clinical measure of disease severity (i.e. World Health Organization functional class, 6-minute walk test or peak $V^{\prime} \mathrm{O}_{2}$ ) reflects an integral measure that is influenced not only by PAH but by comorbidities as well. Because of the small number of patients with underlying connective tissue disease we are unable to comment on the relationship between autonomic control and pulmonary gas exchange variables. However, exclusion of the nonidiopathic $\mathrm{PAH}$ patients did not significantly alter the results of our study.

For logistical reasons we had to use different exercise protocols (treadmill versus bicycle) at the two study sites, which may have confounded our results. This would, however, have made it more unlikely to find a relationship between peak $V^{\prime} \mathrm{O}_{2}$ and autonomic control and should not bias the study towards a false-positive finding. Furthermore, the difference in peak $V^{\prime} \mathrm{O}_{2}$ in $\mathrm{PAH}$ patients appears to be very small when different exercise protocols were used [34].

Finally, although none of the patients had been treated with a $\beta$-blocker, medication may have influenced our measurements of CANA. There are data to suggest effects of endothelin-1 antagonism, digoxin and prostanoids on autonomic function [35-37], which may confound the correlation of measures of CANA and disease severity. This should be taken into account if serial measurements, in particular, are taken with overlapping changes in medication.

\section{Conclusion}

In patients with $\mathrm{PAH}$ there is profound alteration of cardiac autonomic control. The observed changes parallel those seen in chronic left heart failure and relate to disease severity. Measurement of cardiac autonomic function may have a role in clinical assessment and risk stratification of patients with PAH.

\section{STATEMENT OF INTEREST}

A statement of interest for C. Opitz can be found at www.erj. ersjournals.com $/ \mathrm{misc} /$ statements.dtl

\section{ACKNOWLEDGEMENTS}

We would like to thank C. Pohl (Dept of Internal Medicne B, ErnstMoritz-Arndt University Greifswald, Greifswald, Germany) for assistance in the data collection. The authors acknowledge the support of the National Institute for Health Research Biomedical Research Centre scheme (London, UK).

\section{REFERENCES}

1 Thenappan T, Shah SJ, Rich S, et al. A USA-based registry for pulmonary arterial hypertension: 1982-2006. Eur Respir J 2007; 30: 1103-1110.

2 Nootens M, Kaufmann E, Rector T, et al. Neurohormonal activation in patients with right ventricular failure from pulmonary hypertension: relation to hemodynamic variables and endothelin levels. J Am Coll Cardiol 1995; 26: 1581-1585.
3 Sakamaki F, Satoh T, Nagaya N, et al. Correlation between severity of pulmonary arterial hypertension and 123I-metaiodobenzylguanidine left ventricular imaging. J Nucl Med 2000; 41: 1127-1133.

4 Leuchte HH, El Nounou M, Tuerpe JC, et al. N-terminal pro-brain natriuretic peptide and renal insufficiency as predictors of mortality in pulmonary hypertension. Chest 2007; 131: 402-409.

5 Shah SJ, Thenappan T, Rich S, et al. Association of serum creatinine with abnormal hemodynamics and mortality in pulmonary arterial hypertension. Circulation 2008; 117: 2475-2483.

6 Galiè N, Torbicki A, Barst R, et al. Guidelines on diagnosis and treatment of pulmonary arterial hypertension. The Task Force on Diagnosis and Treatment of Pulmonary Arterial Hypertension of the European Society of Cardiology. Eur Heart J 2004; 25: 2243-2278.

7 Hoeper MM, Markevych I, Spiekerkoetter E, et al. Goal-oriented treatment and combination therapy for pulmonary arterial hypertension. Eur Respir J 2005; 26: 858-863.

8 Velez-Roa S, Ciarka A, Najem B, et al. Increased sympathetic nerve activity in pulmonary artery hypertension. Circulation 2004; 110 1308-1312.

9 Ciarka A, Vachièry JL, Houssière A, et al. Atrial septostomy decreases sympathetic overactivity in pulmonary arterial hypertension. Chest 2007; 131: 1831-1837.

10 Eckberg DL, Drabinsky M, Braunwald E. Defective cardiac parasympathetic control in patients with heart disease. $N$ Engl J Med 1971; 285: 877-883.

11 Kleiger RE, Miller JP, Bigger JT Jr, et al. Decreased heart rate variability and its association with increased mortality after acute myocardial infarction. Am J Cardiol 1987; 59: 256-262.

12 van de Borne $\mathrm{P}$, Montano N, Pagani M, et al. Absence of lowfrequency variability of sympathetic nerve activity in severe heart failure. Circulation 1997; 95: 1449-1454.

13 Ponikowski P, Anker SD, Chua TP, et al. Depressed heart rate variability as an independent predictor of death in chronic congestive heart failure secondary to ischemic or idiopathic dilated cardiomyopathy. Am J Cardiol 1997; 79: 1645-1650.

14 Sanyal SN, Ono K. Derangement of autonomic nerve control in rat with right ventricular failure. Pathophysiology 2002; 8: 197-203.

15 Fauchier L, Babuty D, Melin A, et al. Heart rate variability in severe right or left heart failure: the role of pulmonary hypertension and resistances. Eur J Heart Fail 2004; 6: 181-185.

16 Rosas-Peralta M, Sandoval-Zárate J, Attie F, et al. Clinical implications and prognostic significance of the study on the circadian variation of heart rate variability in patients with severe pulmonary hypertension. Gac Med Mex 2006; 142: 19-28.

17 Beaver WL, Wasserman K, Whipp BJ. A new method for detecting anaerobic threshold by gas exchange. J Appl Physiol 1986; 60: 2020-2027.

18 Wensel R, Opitz CF, Ewert R, et al. Effects of iloprost inhalation on exercise capacity and ventilatory efficiency in patients with primary pulmonary hypertension. Circulation 2000; 101: 2388-2392.

19 Davies LC, Francis D, Jurák P, et al. Reproducibility of methods for assessing baroreflex sensitivity in normal controls and in patients with chronic heart failure. Clin Sci (Lond) 1999; 97: 515-522.

20 Heart rate variability: standards of measurement, physiological interpretation and clinical use. Task Force of the European Society of Cardiology and the North American Society of Pacing and Electrophysiology. Circulation 1996; 93: 1043-1065.

21 Ponikowski P, Chua TP, Piepoli M, et al. Augmented peripheral chemosensitivity as a potential input to baroreflex impairment and autonomic imbalance in chronic heart failure. Circulation 1997; 96: 2586-2594.

22 Sun XG, Hansen JE, Oudiz RJ, et al. Gas exchange detection of exercise-induced right-to-left shunt in patients with primary pulmonary hypertension. Circulation 2002; 105: 54-60.

23 Wensel R, Opitz CF, Anker SD, et al. Assessment of survival in patients with primary pulmonary hypertension: importance of cardiopulmonary exercise testing. Circulation 2002; 106: 319-324. 
24 Frenneaux MP. Autonomic changes in patients with heart failure and in post-myocardial infarction patients. Heart 2004; 90: $1248-1255$.

25 Casadei B, Cochrane S, Johnston J, et al. Pitfalls in the interpretation of spectral analysis of the heart rate variability during exercise in humans. Acta Physiol Scand 1995; 153: 125-131.

26 Sleight P, La Rovere MT, Mortara A, et al. Physiology and pathophysiology of heart rate and blood pressure variability in humans: is power spectral analysis largely an index of baroreflex gain? Clin Sci (Lond) 1995; 88: 103-109.

27 Davos CH, Francis DP, Leenarts MF, et al. Global impairment of cardiac autonomic nervous activity late after the Fontan operation. Circulation 2003; 108: Suppl. 1, II180-II185.

28 Davos CH, Davlouros PA, Wensel R, et al. Global impairment of cardiac autonomic nervous activity late after repair of tetralogy of Fallot. Circulation 2002; 106: Suppl. 1, I69-I75.

29 Piepoli M, Clark AL, Volterrani M, et al. Contribution of muscle afferents to the hemodynamic, autonomic, and ventilatory responses to exercise in patients with chronic heart failure: effects of physical training. Circulation 1996; 93: 940-952.

30 Swedberg K, Cleland J, Dargie H, et al. Guidelines for the diagnosis and treatment of chronic heart failure: executive summary (update 2005): The Task Force for the Diagnosis and
Treatment of Chronic Heart Failure of the European Society of Cardiology. Eur Heart J 2005; 26: 1115-1140.

31 Sun XG, Hansen JE, Oudiz RJ, et al. Exercise pathophysiology in patients with primary pulmonary hypertension. Circulation 2001; 104: 429-435.

32 D'Alonzo GE, Gianotti LA, Pohil RL, et al. Comparison of progressive exercise performance of normal subjects and patients with primary pulmonary hypertension. Chest 1987; 92: 57-62.

33 MacGregor AJ, Canavan R, Knight C, et al. Pulmonary hypertension in systemic sclerosis: risk factors for progression and consequences for survival. Rheumatology (Oxford), 40: 453-459.

34 Gläser S, Lodziewski S, Koch B, et al. Influence of the incremental step size in work rate on exercise response and gas exchange in patients with pulmonary hypertension. BMC Pulm Med, 8: 3.

35 Liu JL, Pliquett RU, Brewer E, et al. Chronic endothelin-1 blockade reduces sympathetic nerve activity in rabbits with heart failure. Am J Physiol Regul Integr Comp Physiol 2001; 280: R1906-R1913.

36 Krum H, Bigger JT Jr, Goldsmith RL, et al. Effect of long-term digoxin therapy on autonomic function in patients with chronic heart failure. J Am Coll Cardiol 1995; 25: 289-294.

37 Panzenbeck MJ, Tan W, Hajdu MA, et al. Intracoronary infusion of prostaglandin I2 attenuates arterial baroreflex control of heart rate in conscious dogs. Circ Res 1988; 63: 860-868. 